\title{
Entwicklung eines hochintegrierten, ortsaufgelösten Impedanzmesssystems zur Anwendung in einem intelligenten Zellkulturgefäß
}

\author{
Jonathan Schächtele, Christian Reis
}

Fraunhofer Institut für Produktionstechnik und Automatisierung, Nobelstr. 12, 70569 Stuttgart

\section{Zusammenfassung}

Am Fraunhofer IPA wird ein intelligentes Zellkulturgefäß für adhärente Zellen entwickelt, in dem online ortsaufgelöste Impedanzmessungen mittels IDES an der Zellkultur möglich sind. Die notwendige Elektronik für Datenaufnahme, Aufbereitung und Übermittlung wird direkt in das Gefäß integriert. Das Gefäß bietet damit die Möglichkeit, Zellkulturen permanent unter kontrollierten Bedingungen zu halten, während Messdaten aufgenommen werden. Die Impedanzmessschaltung wurde bereits als Testmuster aufgebaut und Versuche an einer Platine mit entsprechenden Elektrodenstrukturen durchgeführt. Die Versuche legen eine Sensitivität für die Zelldichte nahe, müssen jedoch noch bestätigt werden. Weitere Größen wir Morphologie und Zelladhäsion sollen später ebenfalls untersucht werden.

\section{Einleitung}

Am Fraunhofer IPA ergab sich im Rahmen eines EU-Projekts die Aufgabe, ein Zellkulturgefäß für adhärente Zellen mit integrierter Sensorik auszustatten, um während der Zellkultivierung online Daten über den Zustand der Zellkultur zu sammeln und in einer Regelschleife zu reagieren. Dabei sollen die Kulturen permanent unter kontrollierten Zellkulturbedingungen gehalten werden und nicht etwa zur optischen Analyse aus dem Inkubator entfernt werden müssen. Dadurch ergibt sich die Möglichkeit einer wesentlich reproduzierbareren Zellkultur.

\section{Stand der Technik und Lösungsansatz}

Zwischen biologischem Material und elektromagnetischen Feldern bestehen vielfältige Wechselwirkungen ([1-3]). Deshalb eignen sich Impedanzmessungen bei verschiedenen Frequenzen, um Informationen über den Zustand von Zellkulturen zu sammeln. Bei adhärenten Zellen haben sich hierzu Kammelektrodenstrukturen (Interdigitated electrode structures: IDES) bewährt, die auf der Wachstumsoberfläche aufgebracht sind (z.B. [4], [5]). Aus den Impedanzmessdaten solcher Elektrodenstrukturen lassen sich unter anderem Informationen über zelldichte, Adhäsion und Zellmorphologie ableiten.

Aufgrund der vielversprechenden Ergebnisse mit Impedanzmessungen aus der Literatur, den vorhandenen theoretischen Modellen ([6], [7]), den vielfältigen Verwertungsmöglichkeiten der Daten und der einfachen Realisierbarkeit der Sensorstrukturen wurde entschieden, eine Sensorik für Impedanzmessungen zu integrieren.

\subsection{Konzept}

Zur Kultivierung großer Mengen adhärenter Zellen kommen Gefäße mit großer Wachstumsoberfläche zum Einsatz. In dieser Anwendung hat das Gefäß eine Wachstumsoberfläche von ca. $6000 \mathrm{~mm}^{2}$. Mit einem einzelnen IDES können aber nur Daten an seiner Position erfasst werden. Die Daten eines einzelnen Sensors, der typischerweise einige Millimeter Kantenlänge besitzt, sind hier deshalb nur bedingt aussagekräftig. 
Deswegen wurde eine Matrix aus IDES, die jeweils einzeln ansprechbar sind, vorgesehen. Die Daten können somit ortsaufgelöst bestimmt werden, wovon man sich erhofft, Informationen über die Konfluenz generieren zu können. Außerdem wird die Elektronik zum Aufnehmen der Daten und zur Speicherung direkt in das Zellkulturgefäß integriert (siehe Bild 1), um den Einfluss der Zuleitungen gering zu halten und auch dann Daten aufzuzeichnen, wenn das Gerät nicht an einer elektrischen Schnittstelle angedockt ist.

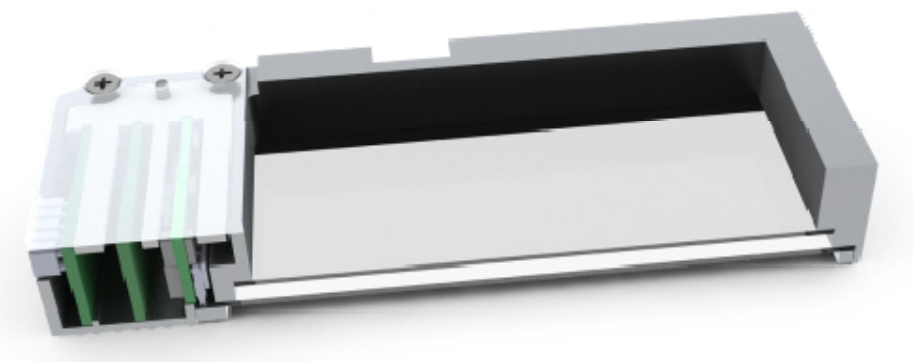

Bild 1 Schnitt durch das Zellkulturgefäß mit integrierter Elektronikbaugruppe

Die Elektronikbaugruppe befindet sich in einem separierten Fach des Bioreaktorgefäßes und kann einfach entnommen werden. Der restliche Bereich des Gefäßes wird sterilisierbar oder als Wegwerfteil ausgeführt.

Die Anforderungen an ein praktisch nutzbares Zellkulturgefäß schließen die Verwendung vorhandener Lösungen zur Impedanzmessung aus: Trotz Sensorik müssen hinreichend große Bereiche in der Zellkulturfläche für die optische Charakterisierung frei bleiben. Die Zellkulturfläche mit integrierten IDES sollte außerdem günstig herzustellen und beschichtbar sein.

Die bisher bekannten IDES-Sensoren für Bioanwendungen bestehen aus Palladium-Elektroden auf Siliziumsubstrat oder Platin-Elektroden auf Saphirsubstrat [8]. Solche Systeme sind jedoch nicht transparent und müssten auf die Wachstumsfläche in komplizierten Prozessschritten aufgebracht und kontaktiert werden. Im Rahmen dieses Projektes sollen als Ziel solche Strukturen mittels Druckverfahren direkt auf eine Polycarbonatscheibe aufgebracht werden, was zusätzlich erhebliche Kostenvorteile verspricht.

\subsection{Messschaltung}

Für die Messschaltung wurde das in Bild 2 gezeigte Konzept erarbeitet. Auf der Zellkulturfläche befinden sich IDES mit einer Größe von $5 \times 5 \mathrm{~mm}$ in einem Raster von $1 \mathrm{~cm}$. Auf der Zellkulturfläche integrierte Kalibierstandards mit bekannter Impedanz machen es möglich, die Einflüsse von Zuleitungen, Multiplexern und Kontaktierungen weitestgehend zu kompensieren.

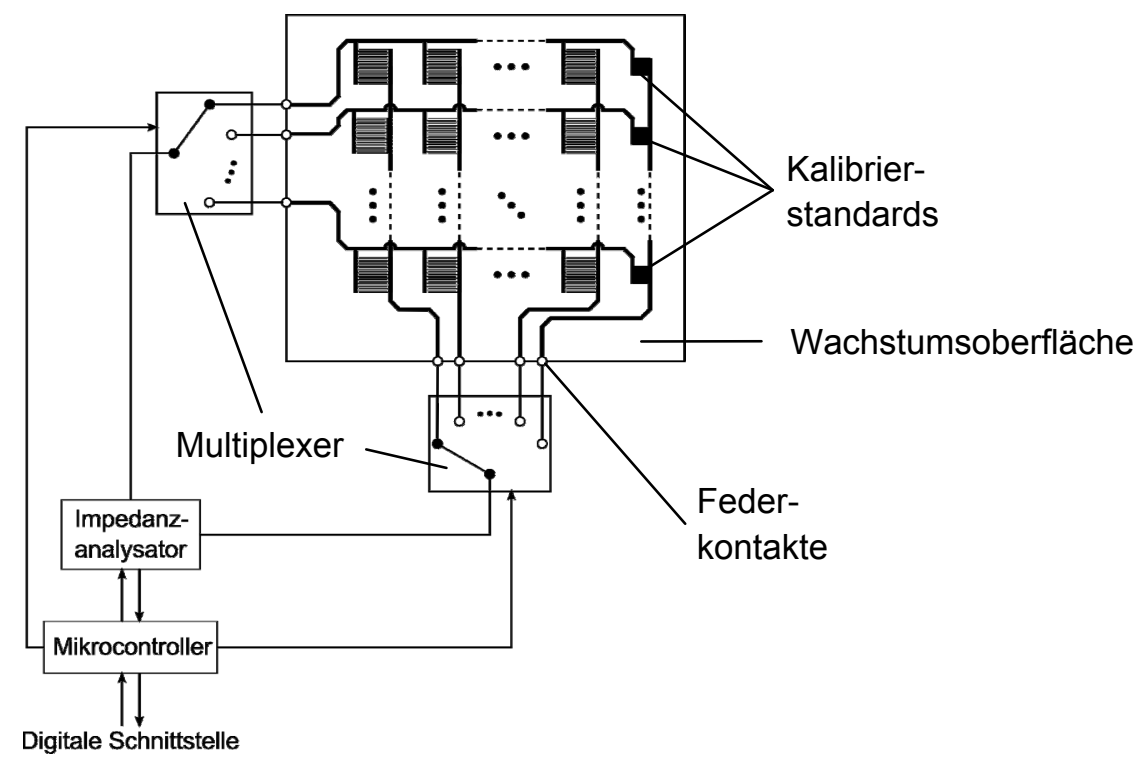

Bild 2 Impedanzsensorik 
Der limitierte Platz im Zellkulturgefäß macht eine hochintegrierte elektrische Baugruppe nötig, die soweit möglich auf integrierte Bausteine setzt. Es konnten Komponenten für die Impedanzmessschaltung gefunden werden, die klein genug sind, um in ein Zellkulturgefäß integriert werden zu können. Als Impedanzanalysator kommt der integrierte Schaltkreis AD5933 von Analog Devices zum Einsatz. Dieser enthält Signalgenerator und A/D-Wandler und führt eine Fouriertransformation auf dem Chip durch, so dass direkt die komplexen Impedanzwerte ausgelesen werden können. Der Frequenzbereich reicht bis $100 \mathrm{kHz}$, was für diese Anwendung ausreichend ist. Es wurde bereits eine elektronische Baugruppe mit Mikrocontroller, Impedanzwandler, Temperaturmessschaltung und Flash-Speicher entworfen, die in das Zellkulturgefäß passt.

\section{Erste Versuche}

Testmuster der Sensorstrukturen und der Auswerteschaltung wurden zunächst auf einer herkömmlichen Platine integriert und mit Zellsuspensionen getestet (siehe Bild 3). Für die Tests kamen 3T3-Fibroplasten in DMEM in unterschiedlichen Konzentrationen zum Einsatz. Die Zellen wurden kurz zuvor aus Zellkulturflaschen mit Trypsin abgelöst, zentrifugiert und in neuem DMEM aufgenommen.
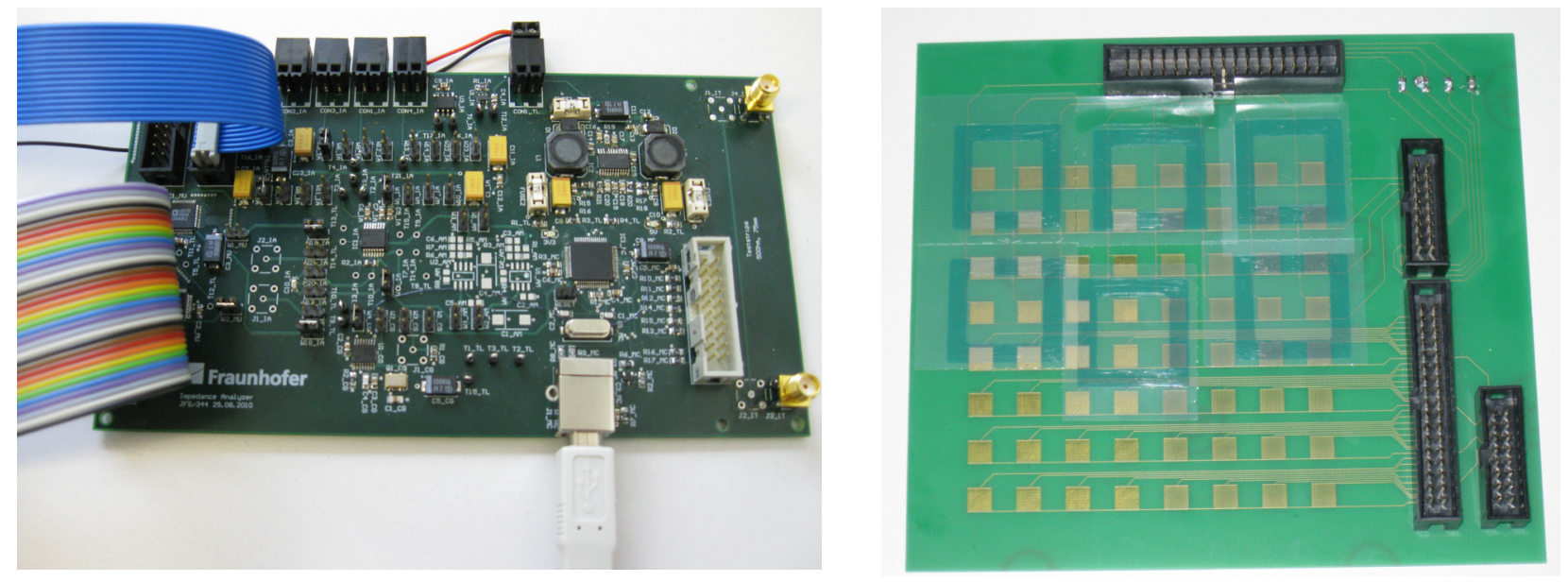

Bild 3 rechts: Testaufbau der Impedanzmessschaltung; links: Platine mit IDES in Matrixschaltung und Zellsuspensionen in aufgeklebten Rahmen

Bei den Versuchen zeigte sich, dass herkömmliche Platinen nicht für den Einsatz in Zellkulturen geeignet sind. Zum einen ist die unmodifizierte Oberfläche nicht zur Zelladhäsion geeignet und zum anderen enthalten sie offensichtlich zytotoxische Substanzen, was dazu führt, dass die Zellen innerhalb weniger Stunden absterben. Da das Material nicht transparent ist, ist eine Korrelation der Impedanzdaten mit den Daten aus optischer Bildgebung nicht möglich.

Bild 4 zeigt Betrag und Phase der gemessenen Admittanz bei einer Frequenz von ca. 10kHz. Die Frequenz wurde entsprechend [4] gewählt. Je nach zu untersuchender Eigenschaft der Zellkultur können andere Frequenzen besser geeignet sein. Es wurden Suspensionen mit ungefähren Zellzahlen von 6500, 32500 und 65000 Zellen in den Volumina der aufgeklebten Rahmen $(65 \mu 1)$ untersucht, sowie reines Medium. Die Messungen lassen einen Zusammenhang zwischen Zelldichte und Betrag der gemessenen Admittanz vermuten, die jedoch noch in weiteren Experimenten bestätigt werden muss. Da bei allen Messungen gleichartige, jedoch nicht dieselben Sensoren verwendet wurden, könnten Unterschiede in den Experimenten auch von Abweichungen an den Sensoren selbst herrühren. Auch Unterschiede in den Kanälen der Multiplexer können Auswirkungen auf die Messergebnisse haben, da solche mit der aktuellen Kalibriermethode nicht kompensiert werden können. 

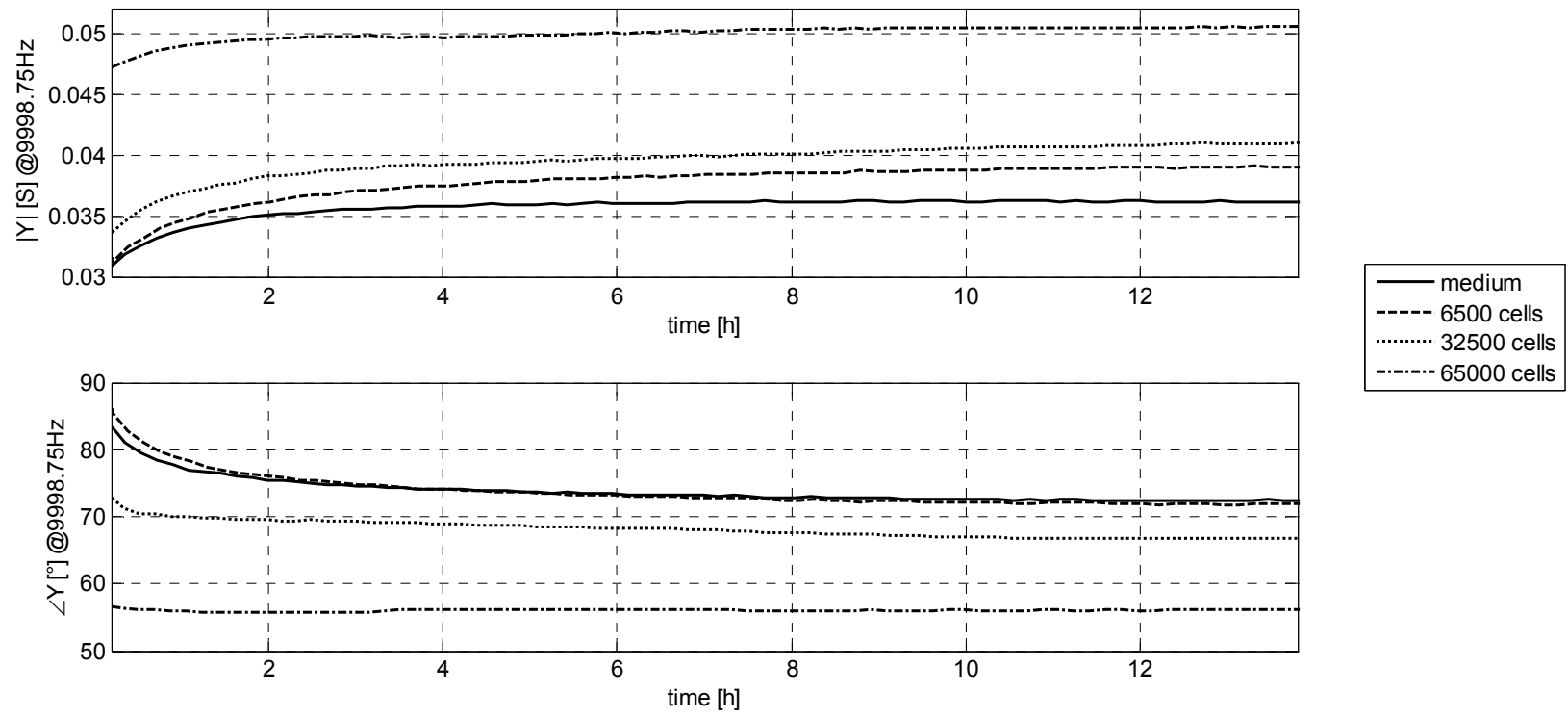

Bild 4 Versuchsergebnisse

\section{Ausblick}

Die elektrische Schaltung muss im Weiteren genau charakterisiert und die gemessen Impedanzen mit den Daten kalibrierter Messgeräte abgeglichen werden, um eine Aussage über ihre Genauigkeit treffen zu können. Weiterhin wird im nächsten Schritt die Sensormatrix mittels Drucktechnik auf einer transparenten Scheibe realisiert, die die Anforderungen an die Biokompatibilität erfüllt. Erst dann können die Daten mit optischen Messungen verglichen werden, was nötig ist, um Aussagen über die interessierenden Größen aus den Impedanzdaten abzuleiten.

\section{Literatur}

[1] H. Schwan, "Dielectric properties of biological tissues and biophysical mechanism of electromagnetic field interaction," ACS Symposium Series, vol. 2, no. 1, 1981.

[2] K. Illinger, "Electromagnetic-field interaction with biological systems in the microwave and farinfrared region," Biological Effects of Nonionizing Radiation, 1981.

[3] C. Justice et al., "Process control in cell culture technology using dielectric spectroscopy.," Biotechnology advances, vol. 29, no. 4, pp. 391-401, 2011.

[4] R. Ehret, W. Baumann, M. Brischwein, A. Schwinde, K. Stegbauer, and B. Wolf, "Monitoring of cellular behaviour by impedance measurements on interdigitated electrode structures.," Biosensors \& bioelectronics, vol. 12, no. 1, pp. 29-41, Jan. 1997.

[5] R. Ehret, W. Baumann, M. Brischwein, A. Schwinde, and B. Wolf, "On-line control of cellular adhesion with impedance measurements using interdigitated electrode structures.," Medical \& biological engineering \& computing, vol. 36, no. 3, pp. 365-70, May. 1998.

[6] X. Huang, D. Nguyen, D. W. Greve, and M. M. Domach, "Simulation of Microelectrode Impedance Changes Due to Cell Growth," IEEE Sensors Journal, vol. 4, no. 5, pp. 576-583, Oct. 2004.

[7] W. Olthuis, W. Streekstra, and P. Bergveld, "Theoretical and experimental determination of cell constants of planar-interdigitated electrolyte conductivity sensors," Sensors and Actuators B: Chemical, vol. 24, no. 1-3, pp. 252-256, Mar. 1995.

[8] B. Wolf, M. Brischwein, W. Baumann, R. Ehret, and M. Kraus, "Monitoring of cellular signalling and metabolism with modular sensor-technique: the PhysioControl-Microsystem (PCM).," Biosensors \& bioelectronics, vol. 13, no. 5, pp. 501-9, Mar. 1998. 